www.richterzeitung.ch

Hans-Jakob Mosimann

\title{
15 Jahre im Rückspiegel - eine Spurensuche
}

Der Blick zurück nach 15 Jahren «Justice - Justiz - Giustizia» gilt der Frage, ob beziehungsweise ab wann die Publikation an welchen Orten wahrgenommen wurde.

Beitragsart: Jubiläum

Zitiervorschlag: Hans-Jakob Mosimann, 15 Jahre im Rückspiegel - eine Spurensuche, in: «Justice - Justiz - Giustizia» 2020/4 
[1] «Justice - Justiz - Giustizia» wird mitunter in Gerichtsurteilen, Bücher und Beiträgen in Fachzeitschriften zitiert. Verschiedene Datenbanken erlauben es, sich ein genaueres Bild der entsprechenden Zitierpraxis zu verschaffen, nämlich Swisslex, die Urteilsdatenbank des Bundesgerichts, sowie bis und mit 2018 auch die Website «www.entscheidsuche.ch». Gesucht wurde mit dem Begriff «Justice - Justiz - Giustizia». Die Treffer wurden sodann nach Häufigkeit ausgewertet, zuerst auf der Zeitachse und sodann anhand zusätzlicher Kriterien. Es handelt sich somit um eine rein quantitative, deskriptive Untersuchung, unter Verzicht auf (spekulative) inhaltliche Schlussfolgerungen.

[2] Die gute Nachricht vorweg: «Justice - Justiz - Giustizia» wurde und wird wahrgenommen, erstmals schon im Jahr 2006 und dann mit steigender und dann sich verstetigender Häufigkeit bis heute (Abbildung 1), wobei das Jahr 2020 nur bis und mit September erfasst ist.

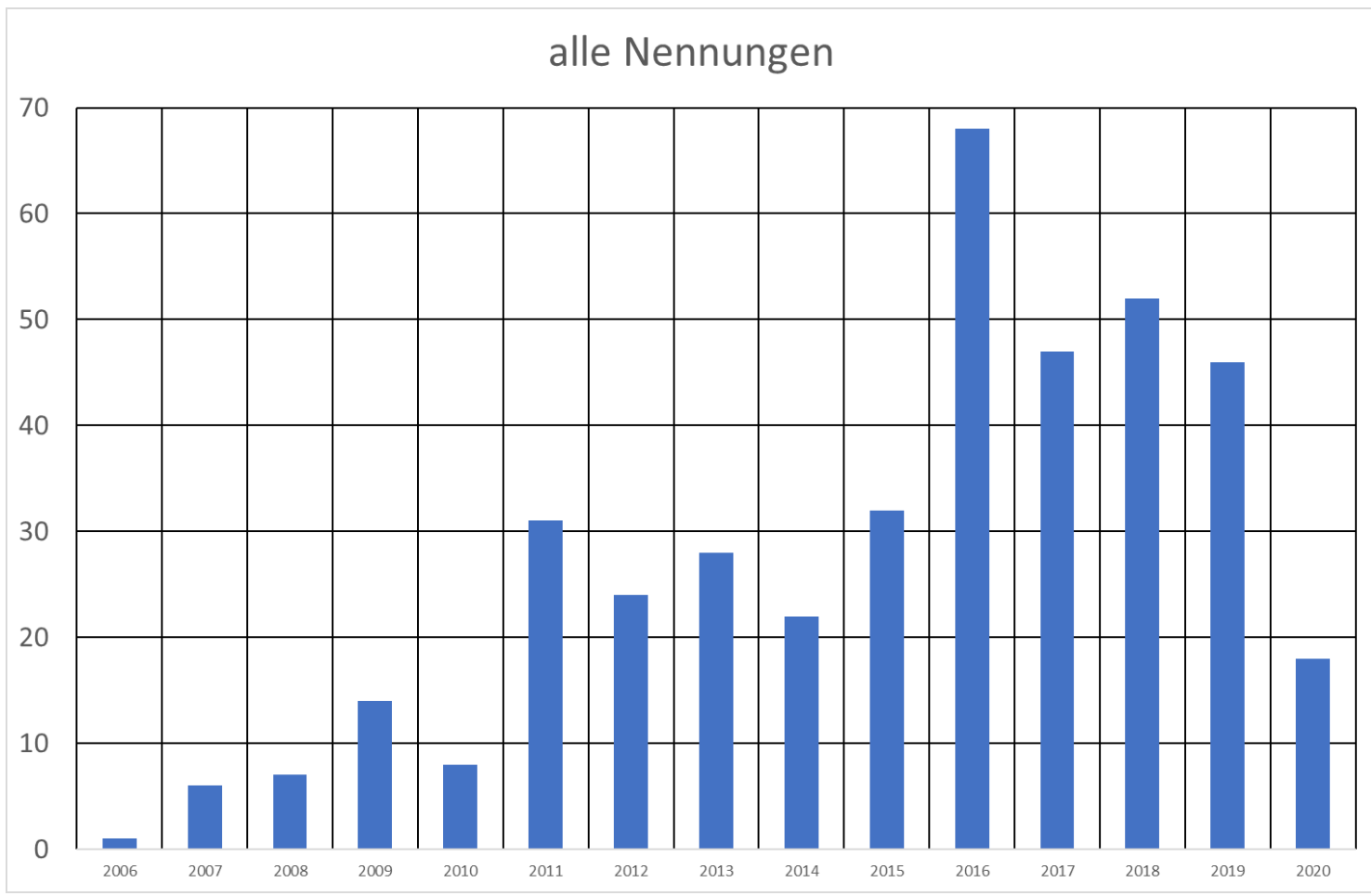

Abbildung 1

[3] Erwähnungen von «Justice - Justiz - Giustizia» finden sich in unterschiedlichen Quellen, nämlich Büchern, Artikeln in Fachzeitschriften und Gerichtsentscheiden (Abbildung 2). Bemerkenswert erscheint die frühe Rezeption in Büchern, die über im Durchschnitt doch rund 12 Nennungen pro Jahr ergibt, und damit sogar diejenige in Fachartikeln (rund neun pro Jahr) übertrifft. Zitate in Gerichtsentscheiden finden sich ab 2007 und bewegen sich in einem Range (von 0) bis fünf oder zehn pro Jahr (2017 dürfte ein Spezialfall, ein «Ausreisser» sein) ${ }^{1}$.

1 Hier hat die Suche sieben Beschlüsse des Obergerichts des Kantons Bern vom 3. November 2017 als Treffer ergeben, die eng verwandte Fälle betrafen. 


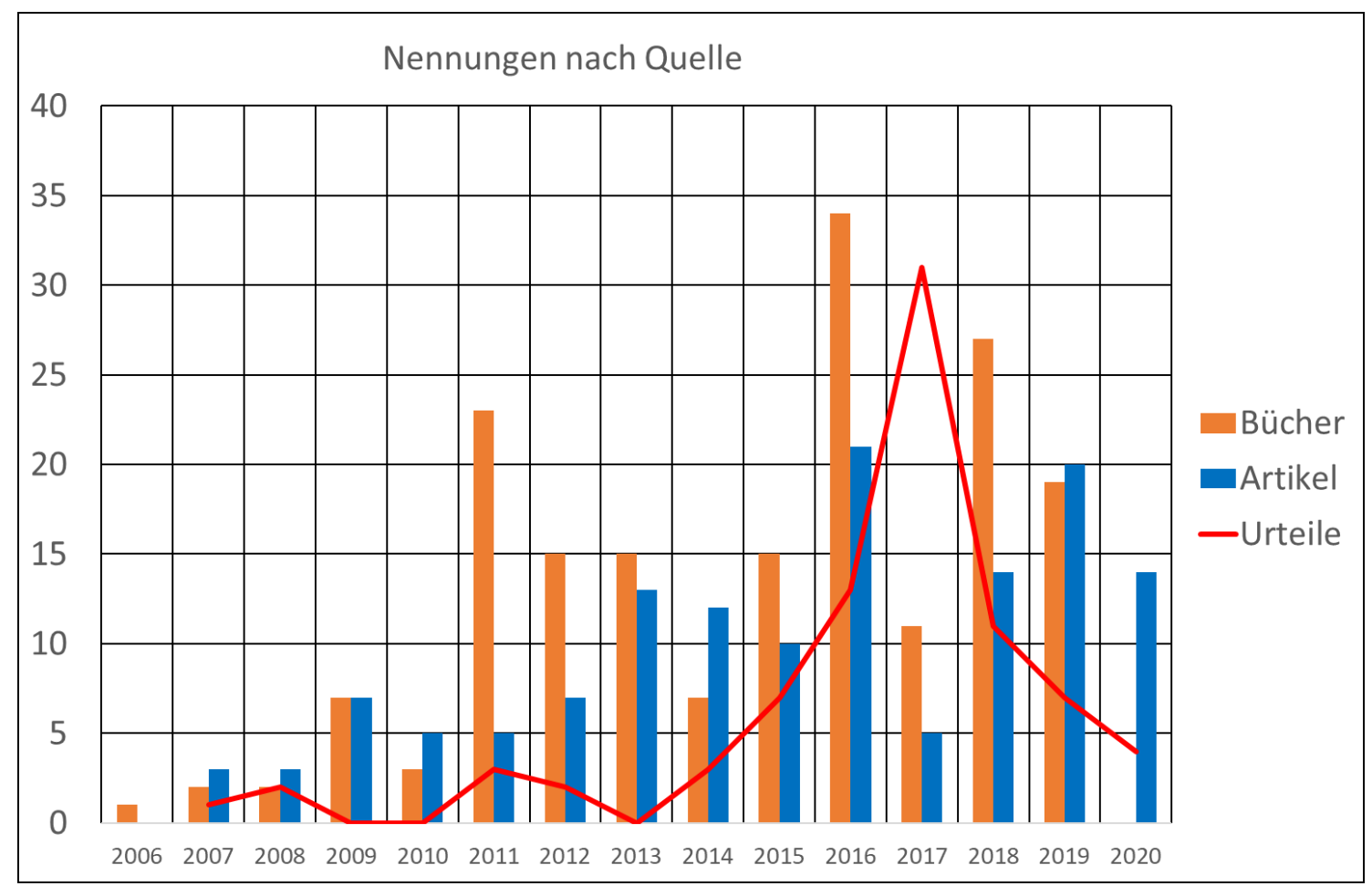

Abbildung 2

[4] Nennungen von «Justice - Justiz - Giustizia» finden sich sowohl in Fachzeitschriften allgemeiner Ausrichtung als auch in solchen, die auf einzelne Rechtsgebiete spezialisiert sind (Abbildung 3). Die Zitate in den All-Themen-Fachzeitschriften (durchschnittlich rund vier pro Jahr) verteilen sich ziemlich gleichmässig über die Jahre, diejenigen in spezialisierten Fachzeitschriften (durchschnittlich rund fünf pro Jahr) schwanken stärker. 


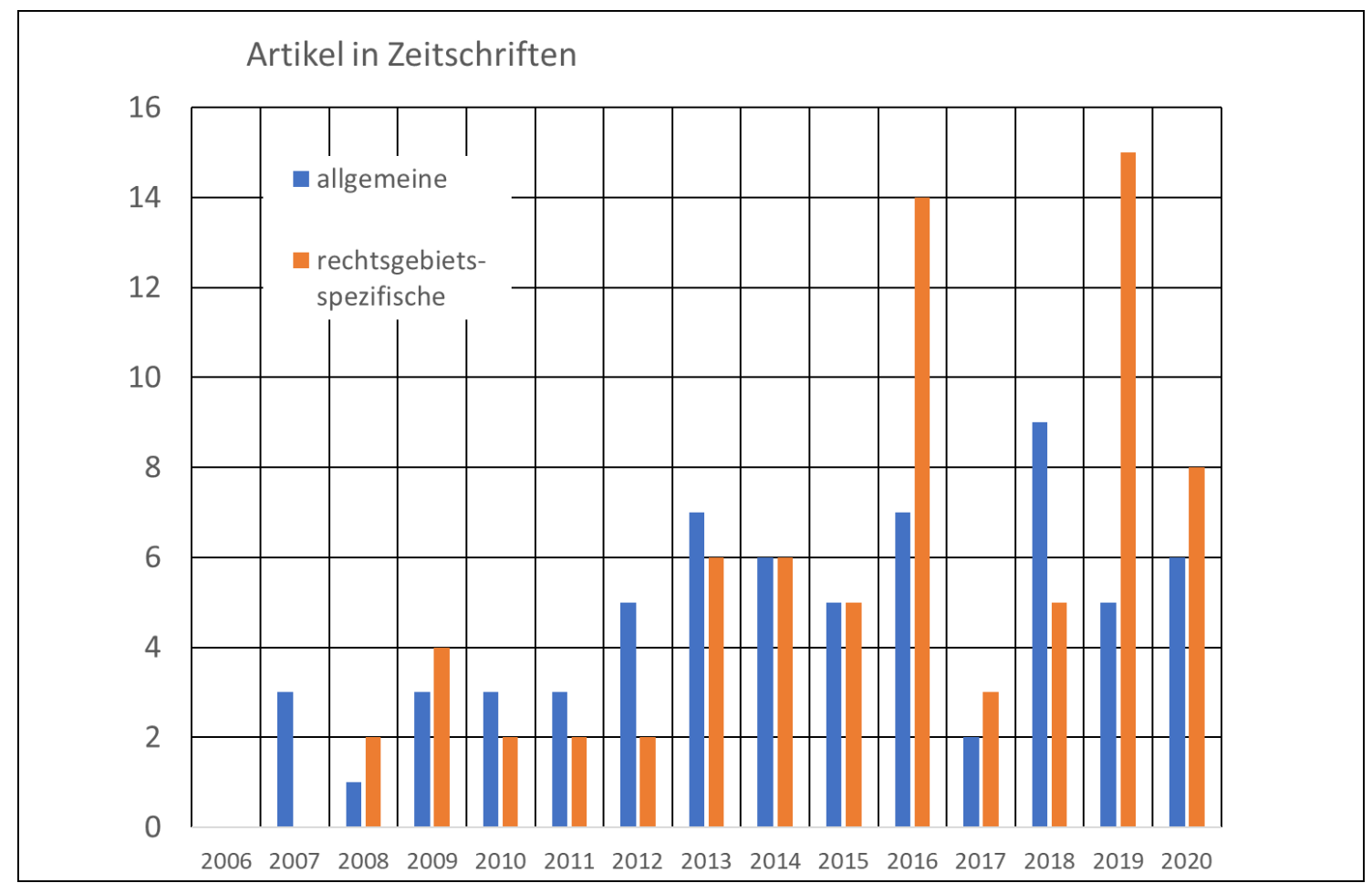

Abbildung 3

[5] Bei den Gerichtsurteilen ist danach zu fragen, welche Gerichte denn «Justice - Justiz - Giustizia» zitiert haben. Angesichts der überblickbaren Gesamtzahl an Nennungen erscheint dabei die Verteilung auf der Zeitachse weniger aussagekräftig als ein Blick über die Zeit hinweg (Abbildung 4). Es finden sich Nennungen in 26 Urteilen des Bundesgerichts, darunter sieben BGE (134 I 238, 137 I 371, 142 I 172, 143 I 194, 143 I 211, 144 III 120 und 146 I 30). Die Erwähnung in BGE 146 I 30 sei als Beispiel herausgegriffen, wo es in Erwägung 2.4 unter anderem heisst: «Demnach stellen die Vergleichsgespräche keinen Schritt auf dem Weg zur gerichtlichen Entscheidung über den Streitgegenstand dar, zumal - wie die Vorinstanz zu Recht bemerkt hat - ihr Inhalt nicht protokolliert wird und einem allfälligen Entscheid des Gerichts nicht zugrunde gelegt werden darf (so etwa LEVI, Der Richter als Vermittler, SJZ 63/1967 S. 255; SCHMID, Vergleichsverhandlungen vor dem Zürcher Handelsgericht, in: Handelsgericht Zürich 1866-2016, Festschrift zum 150. Jubiläum, 2016, S. 248 und 250 f.; vgl. zur Handhabung an den kantonalen Gerichten SCHWEIZER, Praxis der Vergleichsverhandlung, Justice - Justiz - Giustizia 2018/4 Rz. 9 und 16).» Noch etwas mehr, nämlich 32 Nennungen entfallen auf Urteile des Bundesverwaltungsgerichts. Dass über die ganzen 15 Jahre hinweg betrachtet lediglich 23 Nennungen in Urteilen kantonaler Gerichte erscheinen, mag auf den ersten Blick überraschen. Möglicherweise spiegelt dies aber eine nicht sehr ausgeprägte Zitierfreudigkeit der kantonalen Gerichte oder, noch wahrscheinlicher, Unterschiede beziehungsweise Zurückhaltung in der elektronischen Publikation von Urteilen (oder deren Erfassung in den öffentlich zugänglichen Datenbanken). 


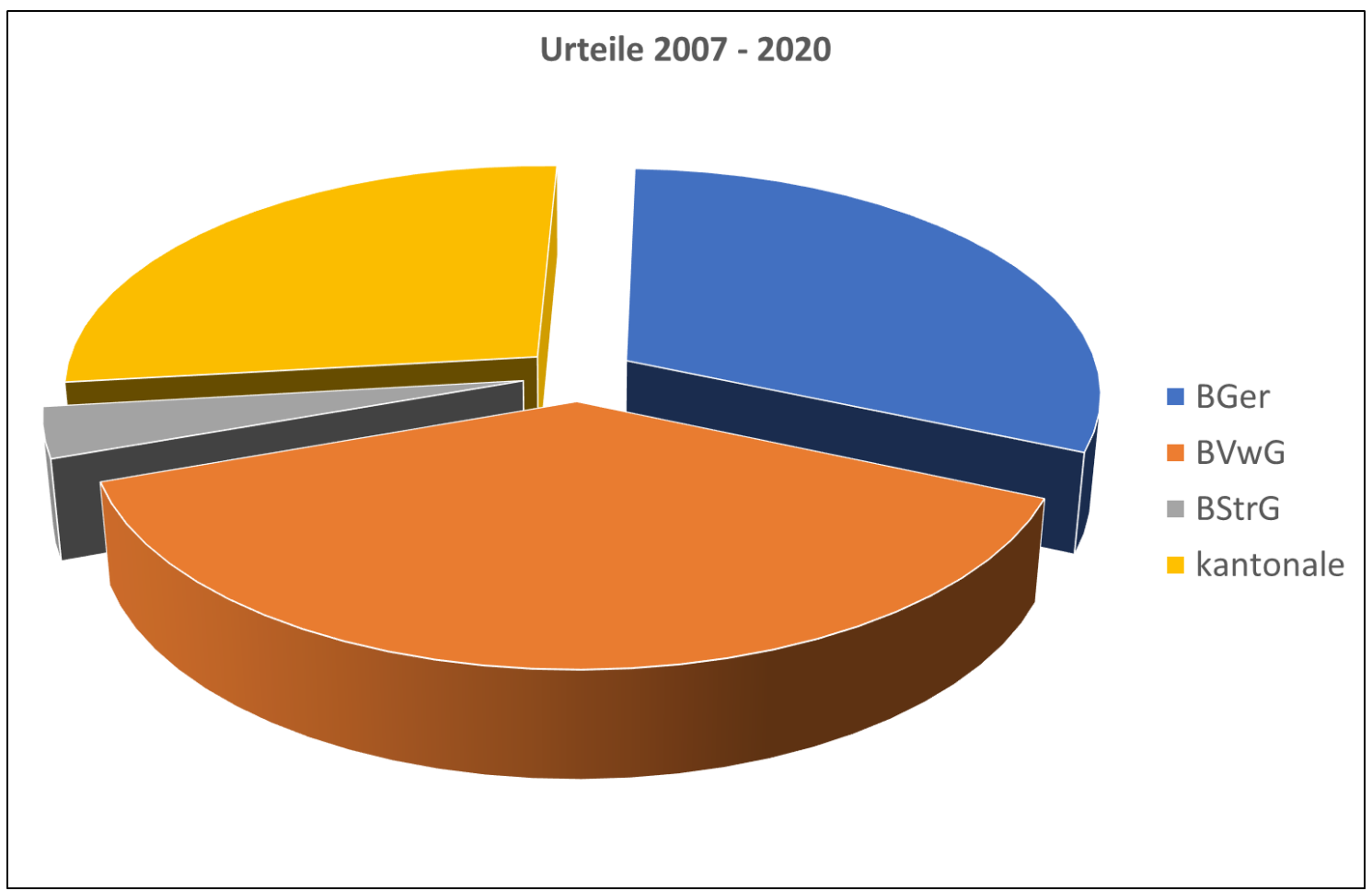

Abbildung 4

Hans-Jаков Mosimann, Dr. iur., Präsident des Sozialversicherungsgerichts des Kantons Zürich und Mitglied des Redaktionsteams der Schweizer Richterzeitung. 